\title{
Pairwise hydrodynamic interactions of synchronized spermatozoa
}

\author{
Benjamin J. Walker $\odot,{ }^{1, *}$ Kenta Ishimoto, ${ }^{2, \dagger}$ and Eamonn A. Gaffney ${ }^{1, \ddagger}$ \\ ${ }^{1}$ Wolfson Centre for Mathematical Biology, Mathematical Institute, University of Oxford, \\ Oxford OX2 6GG, United Kingdom \\ ${ }^{2}$ Research Institute for Mathematical Sciences, Kyoto University, Kyoto, 606-8502, Japan
}

(Received 1 June 2019; published 6 September 2019)

\begin{abstract}
The journey of mammalian spermatozoa in nature is well known to be reliant on their individual motility. Often swimming in crowded microenvironments, the progress of any single swimmer is likely dependent on their interactions with other nearby swimmers. While the complex dynamics of lone spermatozoa have been well-studied, the detailed effects of hydrodynamic interactions between neighbors remain unclear, with inherent nonlinearity in the pairwise dynamics and potential dependence on the details of swimmer morphology. In this study, we will attempt to elucidate the pairwise swimming behaviors of virtual spermatozoa, forming a computational representation of an unbound swimming pair and evaluating the details of their interactions via a high-accuracy boundary element method. We have explored extensive regions of parameter space to determine the pairwise interactions of synchronized spermatozoa, with synchronized swimmers often being noted in experimental observations, and have found that two-dimensional reduced autonomous dynamical systems capture the anisotropic nature of the swimming speed and stability arising from near-field hydrodynamic interactions. Focusing on two initial configurations of spermatozoa, namely those with swimmers located side-by-side or above and below one another, we have found that side-by-side cells attract each other, and the trajectories in the phase plane are well captured by a recently proposed coarse-graining method of microswimmer dynamics via superposed regularized Stokeslets. In contrast, the abovebelow pair exhibit a remarkable stable pairwise swimming behavior, corresponding to a stable configuration of the plane autonomous system with swimmers lying approximately parallel to one another. At further reduced swimmer separations, we additionally observe a marked increase in swimming velocity over individual swimmers in the bulk, potentially suggesting a competitive advantage to cooperative swimming. These latter observations are not captured by the coarse-grained regularized Stokeslet modeling or simple singularity representations, emphasizing the complexity of near-field cell-cell hydrodynamic interactions and their inherent anisotropy.
\end{abstract}

DOI: 10.1103/PhysRevFluids.4.093101

\section{INTRODUCTION}

Rarely found in isolation, mammalian spermatozoa commonly swim as part of large cell populations in a variety of artificial and biological microenvironments [1-4]. Indeed, a single human spermatozoon can be one of hundreds of millions of individuals in a single fertile ejaculate [5]. Despite the relevance of such circumstances to areas of scientific research such as fertility

\footnotetext{
*Author to whom all correspondence should be addressed: benjamin.walker@maths.ox.ac.uk

†ishimoto@kurims.kyoto-u.ac.jp

†affney@maths.ox.ac.uk
} 
diagnostics, where an understanding of the collective behaviors of spermatozoa may be applicable to assisted reproductive therapies and enable more efficient experimentation [6-8], many investigations have considered only the behaviors of a lone spermatozoon. Such studies range from examining the motion of individual swimmers in nontrivial background flows to exploring their behavior in confined geometries [9-14]. However, there have been comparatively few theoretical studies that have considered the collective behaviors of multiple spermatozoa, recent examples being the large-scale numerical simulations of Schoeller and Keaveny [15] and Yang et al. [16], with notable additional work pertaining to swimming in two dimensions, though there remains significant scope for investigating the complex near-field interactions of swimmers in detail. The latter investigations include classical two-dimensional swimming sheet models, introduced in the classical study of Taylor [17] and used later by Fauci and McDonald [13] to study the interactions of model spermatozoa via the immersed boundary method with two-dimensional hydrodynamics. Restricted to motion in a plane, but capturing three-dimensional hydrodynamics, Yang et al. [18] simulated swimmer interactions via multiparticle collision dynamics, utilizing a simple bead model of spermatozoa. A key result of their study was the observation of hydrodynamic attraction between two neighboring spermatozoa, affirmed by the computations of Simons et al. [19] and Llopis et al. [20]. These studies allowed for swimmer motion in three dimensions, and they were based on regularized Stokeslet slender body theory and bead and spring models, respectively, neglecting potential hydrodynamic effects of the swimmer cell body that may not be captured by these methodologies. Interactions between swimmers and boundaries have previously been reported to be highly sensitive to swimmer morphology [21,22], thus it is not clear a priori that the pairwise interactions of spermatozoa will be independent of their geometry. Hence, as a first objective of this study, we will aim to elucidate any behaviors of attraction and repulsion of neighboring spermatozoa that are not bound without confining the cell swimming dynamics to a plane, utilizing a high-accuracy boundary element method to capture the swimmer cell body.

Studying swimmers in configurations directly above or beside one another and considering elastohydrodynamics via a bead-and-spring model, Llopis et al. [20] found that pairwise swimming was unstable for their model swimmers. In addition to remarking on the attraction and repulsion of their driven bead chains, Llopis et al. [20] note a surprising dependence of swimming speed on swimmer configuration, with swimming speed increasing only for swimmers directly above one another, and decreasing for side-by-side swimmers. The experimental study of Woolley et al. [23] concerning bovine spermatozoa supports this conclusion that the relative swimmer position has an impact on changes to the speed of progression effected by proximity, though a detailed theoretical exploration of this result, accounting for complex hydrodynamics and spermatozoan geometry, is, to the best of our knowledge, lacking. Thus, as a second objective of this study, we will attempt to determine the effects of swimmer configuration on swimming speed from a kinematic representation of spermatozoa, in particular noting if the inclusion of the nontrivial geometry of human spermatozoa invalidates qualitative conclusions drawn from simpler models.

Also significant to the interaction of nearby spermatozoa is the phenomenon of flagellar synchronization, in which the coupling of hydrodynamics and filament mechanics can result in outof-phase beating swimmers rapidly adopting a common beat pattern and phase. Synchronization has been observed in various flagellated organisms [23-25], with extensive theoretical study supporting the role of hydrodynamics in filament synchronization [18,26-30]. However, in this study we will consider spermatozoa swimming in very close proximity, with separations typically being less than one cell length, and hence, we will assume throughout that flagellar beating has been synchronized. In turn, this circumvents the need for elastohydrodynamic calculations, which generally suffer from severe numerical stiffness, preventing a detailed exploration of the parameter space associated with the numerous degrees of freedom characterizing the relative motion of two interacting sperm cells, while exploring extensive regions of parameter space underpins the aim of this work.

There has been significant study pertaining to the collective behaviors of swimmers, ranging from the characteristics of bacterial suspensions to active colloidal particles [31-37]. The flow field surrounding an individual spermatozoon is often approximated by that of the force dipole, with 
constant singularity strength of a pusher-type swimmer [38]. The pairwise interactions between such simple model swimmers results in hydrodynamic attraction irrespective of the configuration of the swimmers, due to the symmetry of the flow field representation, though this may not be realistic due to the noted dependence of swimming on cell morphology [39]. Recently, a theoretical coarse-grained approach for the simulation of swimmer populations was suggested by Ishimoto et al. [40] and later implemented by Ishimoto and Gaffney [41], seeking to refine the most basic singularity representation of a spermatozoon. This methodology aims to approximate the flow field induced by a lone swimmer by a small number of regularized singularities [42] with time-varying coefficients for use in large-scale simulations, seeking to improve upon simple far-field singularity representations. Ishimoto and Gaffney [41] simulated a population of spermatozoa moving in two dimensions, with the coarse-grained model well-distinguishing between spermatozoan clustering behaviors emergent from the prescription of different flagellar waveforms, with detailed waveforms representing those that may be observed in spermatozoa in various rheological media. Further, a time-averaged representation of the swimmers fails to reproduce such qualitative differences in the population-level dynamics, highlighting the significance of temporally fluctuating hydrodynamic interactions in the collective behavior of spermatozoa. The efficacy of utilizing their approach in order to qualitatively capture short-range swimmer-swimmer interactions is unknown, as is the ability of the methodology to approximate dynamics in three spatial dimensions. Therefore, as an additional objective of this study, we will evaluate the success of this approach when it is applied to three-dimensional motion, comparing against boundary element computations and ascertaining the degree of qualitative agreement between the two models. As a final objective, we will aim to interpret the computational results of the coarse-grained model in terms of fundamental singularity solutions of Stokes equations, assessing the utility and accuracy of such simple descriptions in approximating swimmer-swimmer interactions.

Hence, in this paper we will examine in detail the hydrodynamic interactions of a pair of virtual spermatozoa, represented by an idealized but representative and commonly used geometry. Prescribing synchronized flagellar kinematics, we will simulate the motion of our virtual swimmers using a high-accuracy boundary element method, aiming to quantify pairwise swimming behaviors in detail, with a particular focus on the direction and speeds of relative progression of the pair. After demonstrating that relative swimmer motion may be reduced to an approximate two-dimensional dynamical system, we analyze such a system to discern long-time swimming behaviors. Further, we will draw a comparison of simulated behaviors with established experimental results, and will determine the level of accuracy at which the simple swimmer representations of Ishimoto and Gaffney [41] predict the interactions of virtual spermatozoa.

\section{METHODS}

\section{A. Virtual spermatozoa}

To model the interactions of two spermatozoa, we introduce the neutrally buoyant virtual kinematic spermatozoon following Ishimoto and Gaffney [10], formed out of an ellipsoidal-like head connected to a long slender flagellum. For the head we adopt the idealized geometry of Smith et al. [14], forming a computational triangular mesh of the surface as parametrized in the Appendix. This is given with respect to a swimmer-fixed frame with right-handed orthogonal coordinate axes $x_{i 1} x_{i 2} x_{i 3}$, where $i \in\{1,2\}$ labels each individual swimmer. These axes are taken such that the $x_{i 1} x_{i 2}$ plane coincides with the plane of the flagellar beat of swimmer $i$, with the corresponding head-tail junction situated at $\left(x_{i 1}, x_{i 2}, x_{i 3}\right)=\mathbf{0}$, where the head-tail junctions have locations $\hat{\boldsymbol{x}}_{0}^{i}$ in the inertial laboratory frame $\hat{x}_{1} \hat{x}_{2} \hat{x}_{3}$. Unit vectors of the swimmer-fixed frames expressed in the laboratory frame are denoted by $\hat{\boldsymbol{e}}_{x_{i j}}$ for swimmer $i \in\{1,2\}$ and direction $j \in\{1,2,3\}$.

Each flagellum is represented by a spherically capped cylinder of radius $0.125 \mu \mathrm{m}$ and length $L_{\mathrm{f}}=56 \mu \mathrm{m}$, a standard scale for human spermatozoa [43], and their motion is prescribed in their respective swimmer-fixed frames. Here we utilize the representative beat pattern of Dresdner and 
Katz [44] as adopted in previous studies of spermatozoon behavior [10], with the flagellar beat parametrized by $s \in\left[0, s^{\star}\right]$ as

$$
x_{i 1}^{\prime}=s, \quad x_{i 2}^{\prime}=(A-B s) \sin \left(k s-\omega t+\psi_{0}(i)\right), \quad x_{i 3}^{\prime}=0,
$$

for wave number $k$, frequency $\omega$, and phase shift $\psi_{0}(i)$, where we take $(A, B)=\left(0.0543 L_{\mathrm{f}}, 0.1087\right)$ for flagellum length $L_{\mathrm{f}}=56 \mu \mathrm{m}$. Here $x_{i 1}^{\prime} x_{i 2}^{\prime} x_{i 3}^{\prime}$ denotes a flagellum-fixed reference frame [14,44], related to the respective swimmer-fixed frame by a time-dependent rotation and translation that ensures that the local flagellum tangent at the base of the flagellum is parallel to $\hat{\boldsymbol{e}}_{x_{i 1}}$, consistent with previous computational studies of human spermatozoa $[10,14]$. The quantity $s^{\star}$ is taken such that the total length of the flagellum is constant, while the angular frequency of the beating is fixed such that $\omega / 2 \pi=14 \mathrm{~Hz}$, in alignment with observations of spermatozoa in Newtonian media [45]. We note that the results that follow are qualitatively independent of this chosen beat frequency, as adjustments to this frequency simply correspond to performing a rescaling in time. Further, throughout we will consider flagellar beating with wave number $k=3 \pi / L_{\mathrm{f}}$ as in Dresdner and Katz [44], though we remark that the presented results are not qualitatively affected by small changes in wave number. With reference to the widely documented synchronization of flagellar beating when swimmers are in close proximity, as remarked in Sec. I, we prescribe $\psi_{0}(1)=\psi_{0}(2)=\psi_{0}$ for some constant phase shift $\psi_{0} \in[0,2 \pi)$, and hereafter assume in this kinematic study that the beating of nearby swimmers is synchronous. For convenience we take $\psi_{0}$ such that the free-space headings of the swimmers are parallel to $\hat{\boldsymbol{e}}_{x_{11}}$ and $\hat{\boldsymbol{e}}_{x_{21}}$, respectively, at time $t=0$. Here and throughout, the term free-space heading refers to the direction of net progression over a beat cycle for a lone swimmer in free-space, and is used as a reference from which we describe the orientation of a swimmer.

\section{B. Governing equations and numerical solution}

The microscale swimming of spermatozoa in an incompressible Newtonian fluid is governed by the three-dimensional Stokes equations, which for pressure $p$ and fluid velocity $\boldsymbol{u}$, expressed in the laboratory frame, are given by

$$
\mu \nabla^{2} \boldsymbol{u}=\nabla p, \quad \nabla \cdot \boldsymbol{u}=\mathbf{0},
$$

where $\mu$ is the viscosity of the fluid, and these equations hold throughout the fluid domain $\Omega$ with boundary $\partial \Omega$. Here we take the fluid domain to be the exterior of the virtual spermatozoa, and apply a no-slip boundary condition on the swimmer surface, additionally enforcing that the fluid velocity decays to zero at spatial infinity in the absence of any nontrivial background flow. Following the boundary element formulation of Pozrikidis [46,47], the solution for the fluid velocity $\boldsymbol{u}$ relative to the laboratory frame at a point $x^{\star}$ on the boundary $\partial \Omega$ is given by

$$
u_{j}\left(\boldsymbol{x}^{\star}\right)=-\frac{1}{4 \pi \mu} \int_{\partial \Omega} G_{i j}\left(\boldsymbol{x}, \boldsymbol{x}^{\star}\right) f_{i}(\boldsymbol{x}) d S(\boldsymbol{x})+\frac{1}{4 \pi} \int_{\partial \Omega}^{\mathrm{PV}} u_{i}(\boldsymbol{x}) T_{i j k}\left(\boldsymbol{x}, \boldsymbol{x}^{\star}\right) n_{k}(\boldsymbol{x}) d S(\boldsymbol{x}),
$$

where $i, j, k \in\{1,2,3\}$ and Einstein summation convention is assumed. Here $G_{i j}$ and $T_{i j k}$ are the standard free-space velocity and stress Green's functions of Stokes flow, which inherently satisfy the condition of far-field decay, $\boldsymbol{n}$ is the surface normal directed into the fluid, $\boldsymbol{f}$ denotes the surface traction, and $\int^{\mathrm{PV}}$ denotes a Cauchy principal-value integral. Application of the no-slip condition at the nodes of the computational mesh representing the surface of the swimmers yields a linear system of equations in the a priori unknown surface tractions $f$ and the cell linear and angular velocities, where the angular velocity of each cell is measured relative to its own head-tail junction. Closure of this underspecified system results from imposing force and torque-free conditions on each swimmer, in addition to removing the gauge freedom in pressure by enforcing that the mean of the normal component of the traction over the domain boundary be zero, without loss of generality.

Given an initial swimmer configuration and prescribed flagellar kinematics, the instantaneous linear and angular velocities of the swimmers are computed and swimmer positions and orientations 


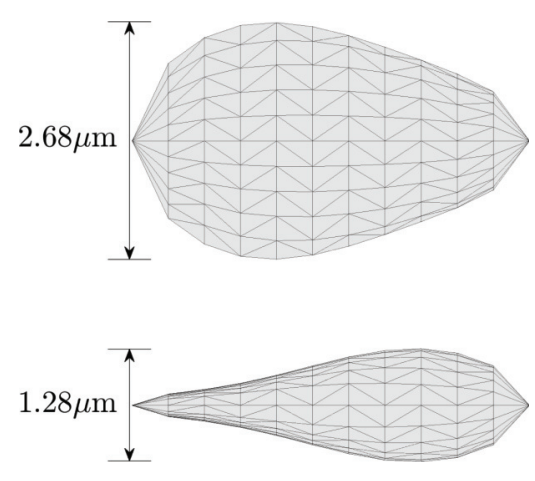

(a)

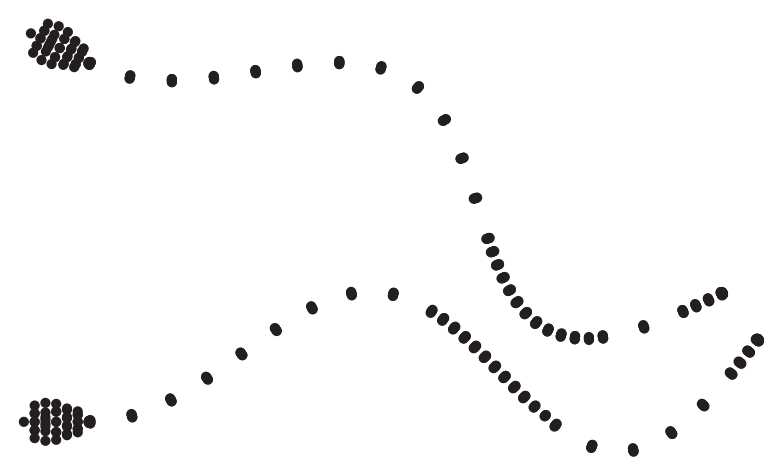

(b)

FIG. 1. Computational meshes representing spermatozoan geometry. (a) Views of a triangular mesh representing the head of a spermatozoon, with vertices created in ellipsoidal bands and equally spaced around each ellipsoidal cross section, following the parametrization given in the Appendix. (b) An example of adaptive meshing based on swimmer proximity, with vertices shown as points and elements omitted. The distal portions of the flagella of both swimmers have been partially remeshed to increase local accuracy while retaining computational efficiency. The mesh is shown at reduced resolution for visual clarity, though the numerous vertices at each cross section of the flagellum are effectively amalgamated into one ellipsoidal marker point due to the resolution of the image. Finally, note that these flagella correspond to a flagellum length of $56 \mu \mathrm{m}$, a standard scale for human spermatozoa [43].

updated via a predictor-corrector scheme, Heun's method, with time step $d t$, which for swimmer position $\hat{\boldsymbol{x}}_{0}^{i}(t)$ and linear velocity $\boldsymbol{U}^{i}(t)$ is given explicitly by

$$
\hat{\boldsymbol{x}}_{0}^{i}(t+d t)=\hat{\boldsymbol{x}}_{0}^{i}(t)+\frac{d t}{2}\left[\boldsymbol{U}^{i}(t)+\boldsymbol{U}^{i}(t+d t)\right] .
$$

In this study, we focus on the hydrodynamic interactions of the swimming pair, and do not include in our model any swimmer-swimmer contact interactions, halting numerical simulations prior to collision, noting that both short-range attractive and repulsive forces are of physical relevance but are typically only modeled phenomenologically $[19,22,48,49]$. Computational meshes consisting of 2848 triangular elements per swimmer are adaptively refined based on swimmer proximity, with quantities linearly interpolated over elements. Example meshes demonstrating swimmer head geometry and proximity-based adaptive meshing are shown in Fig. 1. The suitability of computational meshes was established by numerical convergence, with numerical procedures being validated against the boundary element methods of our previous studies [10,22]. The time step $d t$ is typically taken such that there are 100 time steps per beating period, though temporal refinement is increased by an order of magnitude when swimmers are in very close proximity.

\section{Swimmer configurations}

The interactions of swimming spermatozoa with synchronized flagellar beats naively form a 13-dimensional system, with six scalar quantities representing the location and orientation of each individual swimmer, in addition to the phase of the shared flagellar beat. Consideration of only their relative motion eliminates six degrees of freedom, though full exploration of even this reduced system is computationally prohibitive. We will consider the motion of swimmers initiated in one of two configurations, termed side-by-side and above-below, similar to the work of Llopis et al. [20] and each of relevance to the wealth of captured videomicroscopy of spermatozoa, in which nearby swimmers commonly share a beat plane or lie closely above one another in the plane of imaging, as seen in the supplemental information of [50,51]. From these initial configurations, swimmer motion 


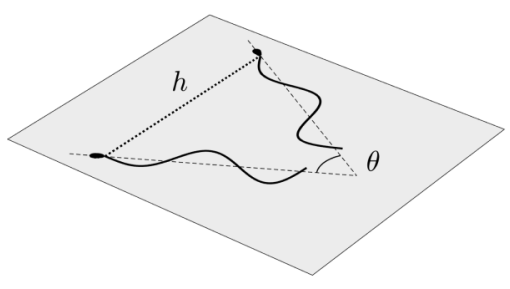

(a)

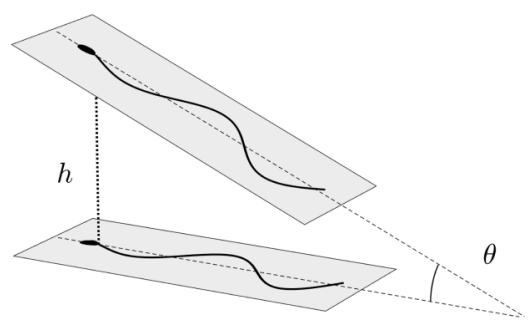

(b)

FIG. 2. Schematic of virtual spermatozoa configurations and accompanying parametrizations, with freespace headings being shown as dashed lines and their separation $h$ represented by a dotted line. (a) Swimmers share a common beat plane in the side-by-side configuration, with $\theta$ defined as the signed planar angle between their free-space headings. (b) In the above-below configuration, the swimmers' relative heading is defined as the signed angle between their beat planes, as shown, where we are making the approximation that the relative motion of the swimmers is expressible solely by the quantities $h$ and $\theta$, justified a posteriori in Secs. IIIC and III B. In both configurations, $\theta=0$ corresponds to the virtual spermatozoa sharing both beat plane orientation and free-space heading, and we adopt the sign convention such that $\theta>0$ denotes spermatozoa facing away from one another.

is simulated unconstrained, allowing for full three-dimensional interactions between swimmers. In Fig. 2(a) we show a side-by-side configuration of swimmers, in which the spermatozoa share a beat plane and their relative motion may be described in part by the separation of their head-tail junctions, $h=\left|\hat{\boldsymbol{x}}_{0}^{1}-\hat{\boldsymbol{x}}_{0}^{2}\right|$, and the relative angle between their free-space headings, $\theta$, where we are neglecting an additional degree of freedom describing their relative in-plane displacement, to be justified a posteriori given initial conditions with swimmers directly beside one another.

In Fig. 2(b) we exemplify the above-below configuration of swimmers, in which virtual spermatozoa beat planes are parallel to each other except for rotations about the swimmer-fixed $x_{i 2}$-axes. Notably $\theta=0$ corresponds to agreement between the free-space headings of the swimmers, with any rotations within the plane of beating being neglected. Indeed, we hypothesize that consideration of such in-plane rotations is of limited physical relevance due to the minimal magnitude of swimmer interactions in this configuration, a hypothesis that we will examine in detail in Sec. III C.

\section{Reduction of behavior to an approximate low-dimensional dynamical system}

To systematically capture the complex behaviors of interacting spermatozoa, we simulate the motion of the two swimmers for a single period of their synchronized flagellar beats, recording their change in separation $h$ and relative heading $\theta$, initially enforcing that the swimmers are directly beside or above each other. We note that the dependence of the relative motion of the swimmers on the initial phase $\psi_{0}$ is expected to be minimal, as is confirmed by numerical simulation, and thus we take $\psi_{0} \equiv 0$ throughout. This effectively reduces the swimming motion to an approximate two-dimensional dynamical system in $h$ and $\theta$, enabling the application of a dynamical systems framework as in Walker et al. [22,52]. Validity of this methodology is established by direct comparison with long-time simulations of swimmer interactions, which highlights in particular that the relative displacement of swimmers along their respective headings does not qualitatively impact the $h-\theta$ dynamics on relevant timescales from initial conditions of zero displacement.

\section{E. Coarse-grained singularity representations of swimmers}

We will attempt to evaluate the accuracy of a recent regularized singularity representation [40,53,54], as used by Ishimoto and Gaffney [41] to simulate population-level behaviors of spermatozoa confined to a plane, in particular considering its natural extension to motion in three 
spatial dimensions. Here we briefly recapitulate the key principles of the methodology, and direct the reader to the original publications $[40,41,53,54]$ for further details.

Given the flow field generated by a swimmer in the absence of any other fluid boundaries or background flows, principal component analysis is used to extract high-variance modes of velocity field. The two most significant modes, which in this case capture $91.6 \%$ of the variance of the flow field, are approximated via a small distribution of regularized Stokeslets [42], yielding a lowdimensional representation of the time-varying flow field associated with a single isolated swimmer that has been validated to reasonable accuracy even in the presence of a planar boundary. This representation, which will be shown graphically in Sec. III E, allows efficient simulation of multiple swimmers in the same domain, with the velocity of any individual swimmer being determined via force and torque-balance equations, for instance with the drag on a given swimmer approximated by the sum of the strengths of its constituent regularized Stokeslets.

\section{RESULTS}

\section{A. Swimming in close proximity is transient for side-by-side spermatozoa}

The long-time motion of two virtual spermatozoa in close proximity in a side-by-side configuration was simulated using the boundary element method over numerous beat cycles, where both swimmers are equipped with the same in-phase flagellar beat and are swimming within the same plane. Simulations from various initial configurations demonstrated that nearby swimming in a side-by-side configuration is transient, with the swimmers either colliding with or deflecting away from one another. Initial separations of the head-tail junctions were sampled in the range $h \in[2.5 \mu \mathrm{m}, 50 \mu \mathrm{m}]$, with the relative angle of free-space progression $\theta$ ranging from $-\pi / 4$ to $\pi / 4$. Typical traces showing the location of the head-tail junction of each swimmer over time are presented in Fig. 3(a) for side-by-side swimming, demonstrating collision and deflection of the swimmers that is dependent on their relative initial configurations.

To systematically classify these behaviors of collision and deflection, we proceed by considering the approximate dynamical system as described in Sec. IID. The resulting phase plane is shown in Fig. 3(b), along with projections of long-time simulations of unconstrained swimmer motion, demonstrating very good agreement between swimmer motion and the approximate reduction to a plane autonomous system. In particular, this agreement validates a posteriori the use of such a phase plane in studying the dynamics of a swimming pair in this configuration, and highlights that any relative motion of the swimmers along their respective axes of progression does not significantly alter their behaviors on reasonable timescales from these initial conditions; indeed, the sample long-time trajectories shown in Fig. 3(a) exemplify that such displacement is minimal. Absent from the plane autonomous system is a stable swimming configuration or limit cycle, meaning that trajectories either result in collision or represent swimmers separating off into the bulk, and thus pairwise side-by-side swimmer motion is transient. Remarkably, we observe from the phase plane that even swimmers initially facing away from one another may eventually collide as the result of proximity-induced reorientation, with the magnitude of this effect decreasing as the separation between their head-tail junctions $h$ increases.

\section{B. Above-below swimming dynamics approach a stable spiral}

Having examined the interactions of swimmers that share a common beat plane, we repeat the phase-plane quantification of pairwise swimmer dynamics for virtual spermatozoa in an abovebelow configuration, with swimmers initially situated directly above one another. The reduced system, shown in Fig. 4, exhibits convergence to a stable spiral in the $h-\theta$ space from a wide range of initial configurations in the phase space, assuming that initially the free-space headings of the swimmers lie within parallel planes and that the virtual spermatozoa start out located directly above one another. With the central fixed point located at $(h, \theta) \approx(19.1 \mu \mathrm{m}, 0.0076 \pi)$, this spiral corresponds to swimmers slowly oscillating about a configuration where they are oriented almost parallel to one another, and represents a stable mode of pairwise swimming in close proximity. The 

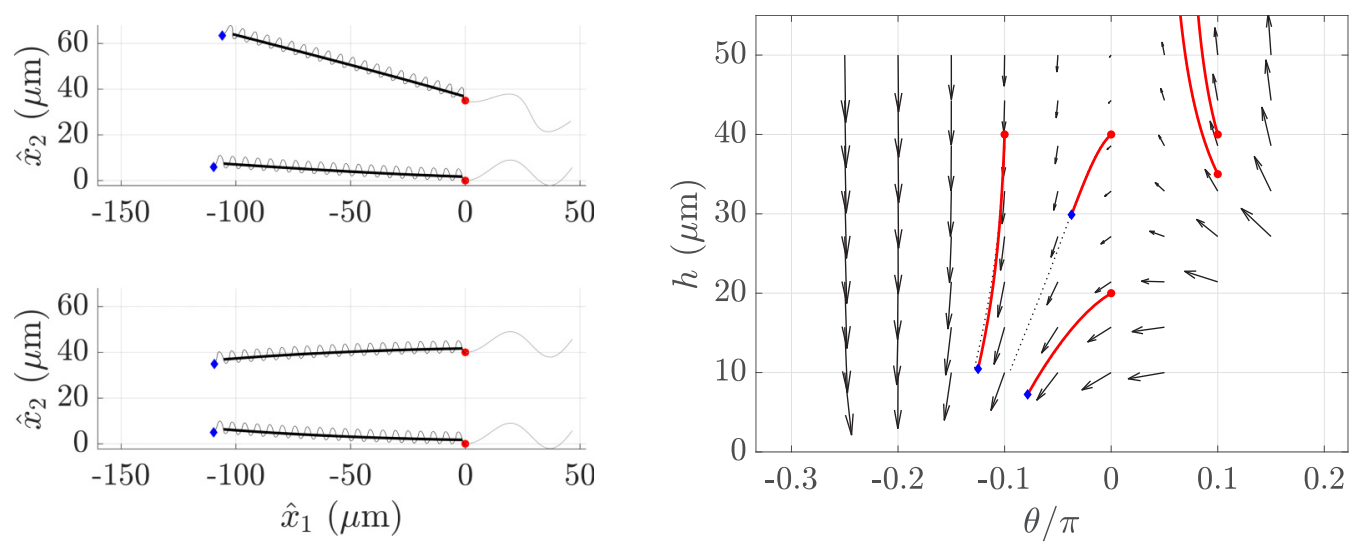

(a)

(b)

FIG. 3. The transient dynamics of side-by-side swimming, with trajectory start and end points shown as red circles and blue diamonds, respectively. (a) Each thin curve shows the positions of the head-tail junctions $\hat{\boldsymbol{x}}_{0}^{i}$ of a pair of spermatozoa in the laboratory frame $\hat{x}_{1} \hat{x}_{2} \hat{x}_{3}$ for side-by-side swimmers with initial conditions $(h, \theta)=(35 \mu \mathrm{m}, 0.1 \pi)$ and $(h, \theta)=(40 \mu \mathrm{m}, 0)$ for the upper and lower panels, respectively, and simulated over 20 periods of the flagellar beat. Averaged swimmer paths are shown as heavy black curves, highlighting separation in the upper panel and convergence of the swimmers in the lower panel. Shown in gray are the swimmers in their original configurations, with the cell bodies omitted here for clarity. (b) Pairwise dynamics in the $h-\theta$ space, with the projections of sample long-time simulations, typically simulated for 20 periods of the flagellar beat, shown as solid red curves. Solutions of the approximate plane autonomous system are shown as dotted curves with the same initial conditions as the long-time simulations, demonstrating very good agreement between the full and reduced systems, serving as a posteriori validation of the reduction to $h-\theta$ space. Further, the dynamics as predicted by the phase plane exhibit no stable pairwise swimming, merely the behaviors of eventual collision and separation off into the bulk, though sperm initially heading away from one another are predicted to sometimes still be on a path that will result in eventual collision.

existence of a stationary point in the $h-\theta$ space may be explained intuitively by a simple balance of hydrodynamic attraction between swimmers and their progression along their free-space headings, noting that swimmers are oriented slightly away from each other in the stable configuration, though it is a priori unclear that such mutual attraction should be present at all.

To validate the existence of this stable swimming behavior in the full dynamics, a number of long-time simulations of unconstrained swimmer motion were performed, with sample projections onto the $h-\theta$ space shown as red curves in Fig. 4. As was the case for side-by-side swimmers, excellent agreement is observed in general between trajectories predicted from the plane autonomous system and those projected from long-time simulations. In particular, a simulation of above-below swimmers beginning in the steady-state configuration predicted by the reduced dynamics demonstrates pairwise swimming behavior corresponding to the stable spiral in the $h-\theta$ space, and it is robust to perturbations out of an initial above-below configuration. Thus, from consideration of a validated reduction of the dynamics to a plane autonomous system, we have identified a stable form of pairwise swimming in virtual spermatozoa, present for swimmers in an above-below configuration and corresponding to approximately parallel motion. Further, and most remarkably, we have observed that this mode of behavior is indeed exhibited in long-time simulations of the full pairwise dynamics.

\section{Timescales of pairwise interaction are configuration-dependent}

In addition to enabling an analysis of the overall behaviors of swimming spermatozoa, identifying collision, deflection, or stable relative motion, the long-time simulations and approximate 


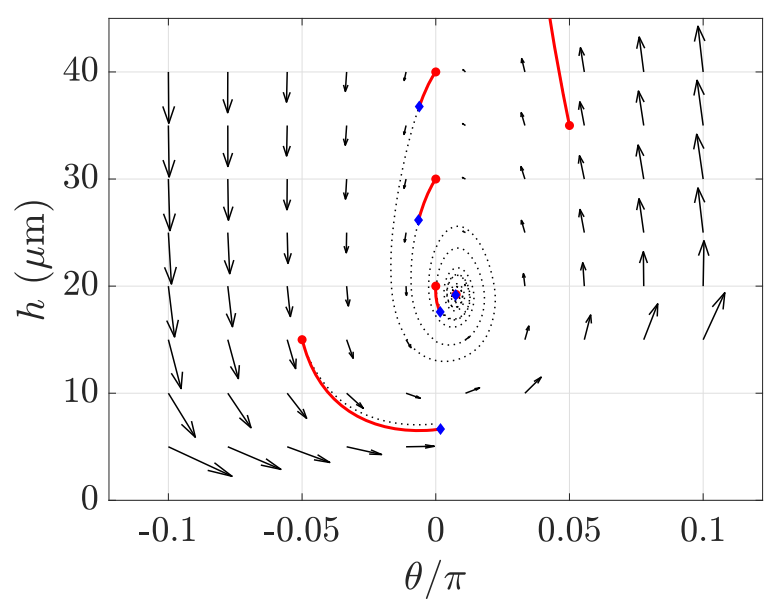

FIG. 4. The approximate dynamics of above-below swimming, highlighting a pairwise swimming mode that corresponds to stable long-time motion in close proximity, represented here by the existence of an attracting stable spiral around $h \approx 19.1 \mu \mathrm{m}, \theta \approx 0.0076 \pi$. Projections of long-time simulations, computed for 20 periods of the flagellar beat and serving as a posteriori validation of the phase plane, are shown as red curves, with start and end points displayed as red circles and blue diamonds, respectively. In particular, a long-time simulation initiated from approximately the stationary point of the autonomous system shows that swimmers indeed undergo negligible relative motion, verifying the existence of a stable swimming configuration. Solutions of the approximate plane autonomous system are shown as dotted curves with the same initial conditions as the long-time simulations, demonstrating very good agreement between the full and reduced systems. Trajectories can be seen to spiral inward, approaching the stationary point on timescales on the order of hundreds of beat cycles.

phase-plane computations of Secs. III A and III B reveal a disparity in timescales between swimmer configurations. While this may be deduced from detailed consideration of the phase planes of Figs. 3 and 4, this is most clearly exemplified by focusing on the relative motion of swimmers in each configuration with $\theta=0$ initially. In Fig. 5 we report the change in separation between their head-tail junctions along with the difference in relative heading over a single beat period as a function of initial separation, as used to generate the profiles of the reduced systems in Secs. III A and III B. From this we note that the changes in both swimmer separation and relative heading over a beat period are significantly greater for side-by-side swimming than for swimmers in an above-below configuration, with the change in heading in particular being approximately an order of magnitude less for above-below swimmers. Hence, we conclude that the hydrodynamic effects of proximity on swimming behavior are most prominent for virtual spermatozoa that share a plane of beating, in contrast to the relatively weak effects experienced by swimmers in an above-below configuration, highlighting a stark anisotropy in the interactions of neighboring swimmers.

In particular, the reduced magnitude of swimmer interactions in the above-below configuration justifies a posteriori our consideration of above-below swimming only where there is no relative rotation within the swimmers' planes of beating. Indeed, were there to be such a relative rotation, virtual spermatozoa may be expected to simply pass over each other and separate with little observable interaction, owing to the noted relative subdominance of out-of-plane hydrodynamic interactions in this configuration.

\section{Effects of proximity on swimming speed are anisotropic and commonly inhibit progression}

With swimming stable in above-below configurations and transient over long timescales for side-by-side swimmers, we consider in more detail the effects of low cell separation on swimmer 

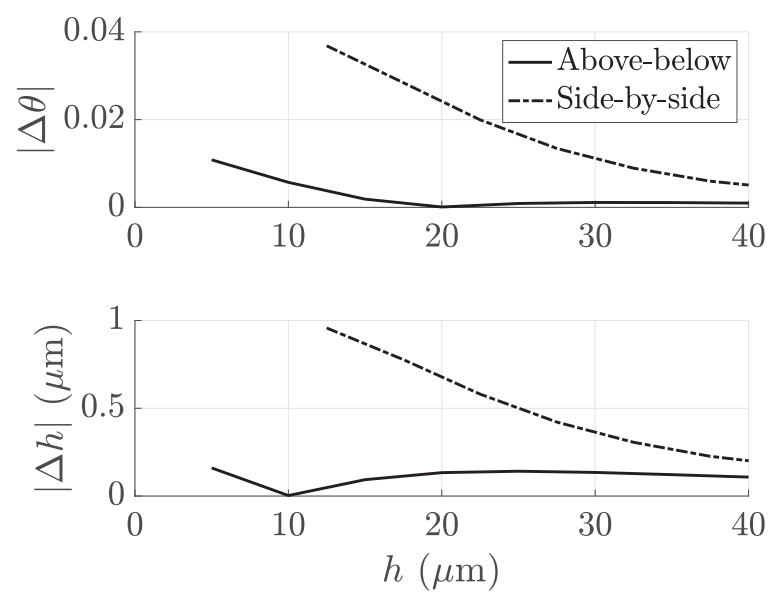

FIG. 5. Change in swimmer separation and relative heading over a single beating period as a function of initial separation $h$, denoted $\Delta h$ and $\Delta \theta$, respectively. Initially the swimmers are situated directly above or beside one another, and with $\theta=0$. Above-below swimmers, shown as solid curves, can be seen to undergo reduced changes in relative heading and separation when compared to side-by-side swimmers, shown as dotdashed curves, highlighting a disparity in the timescales of relative motion between swimmer configurations.

progression. In particular, for swimmer pairs in each of the side-by-side and above-below configurations, we compute the average linear velocity of the swimmers over a single beating period, whereas above the flagellar beats are assumed to be in synchrony.

Considering first the case of side-by-side swimming, the swimming speed being shown as a dot-dashed curve in Fig. 6, we see that a reduction in swimmer separation yields a substantially reduced average swimming speed, with each swimmer inhibiting the progression of the other.

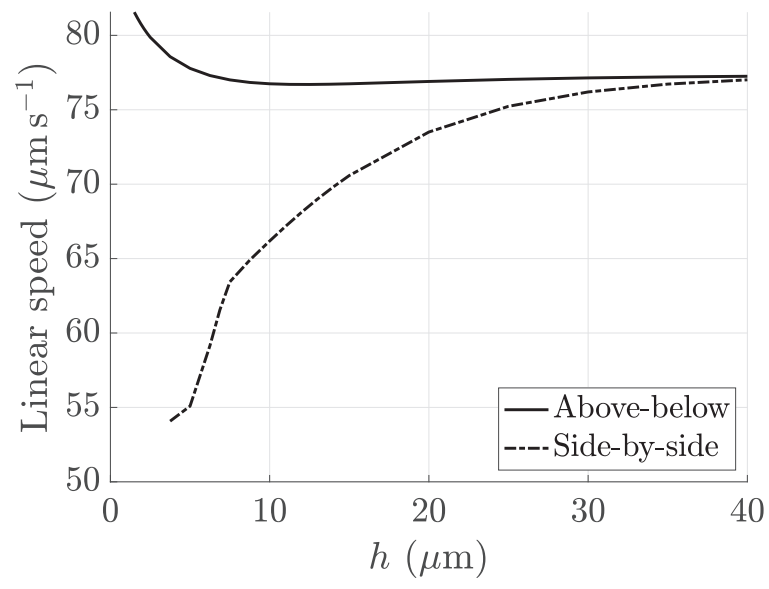

FIG. 6. The linear velocity of a swimmer over a single beating period as a function of separation, $h$, in both above-below and side-by-side configurations. With swimmers initially directly above/beside one another, and shown here with $\theta=0$, observed for both configurations is a reduction in swimming speed with separation between swimmer head-tail junctions, though the magnitude of such reduction is notably greater in the case of side-by-side swimming than for above-below swimmers, the change in the latter being difficult to observe on the scale of the above plot. At further-reduced separations, below approximately $10 \mu \mathrm{m}$, remarkably, virtual spermatozoa in an above-below configuration can be seen to increase in speed, surpassing even their free-space swimming speed of approximately $77 \mu \mathrm{m} \mathrm{s}^{-1}$. 


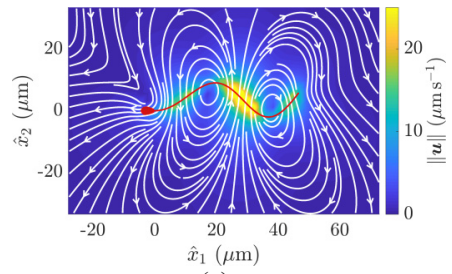

(a)

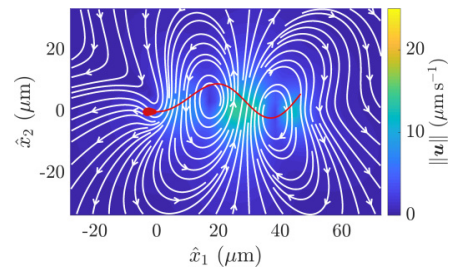

(b)

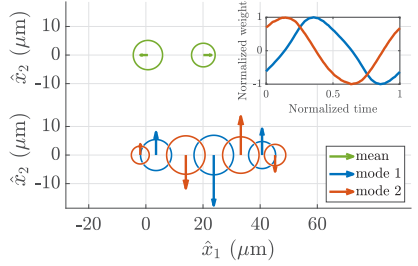

(c)

FIG. 7. Instantaneous flow field in the plane of beating for an individual virtual spermatozoon in the laboratory frame $\hat{x}_{1} \hat{x}_{2} \hat{x}_{3}$, with the swimmer shown in red and situated at the origin of the laboratory frame. The flow field as computed by the boundary element method is given in (a), with the corresponding regularized singularity representation flow field shown in (b). (c) An illustration of the low-dimensional regularized singularity representation, comprised of the lowest two PCA modes and the mean over a beating period, with arrow size and direction corresponding to the strength and direction of the regularized Stokeslets. The circle size corresponds to the regularization parameter taken for each regularized Stokeslet, and inset is the normalized weighting of each of the PCA modes over a single beating period. Each regularized Stokeslet lies on the line $\hat{x}_{2}=0$ by the symmetry of the flagellar beat, with those corresponding to the mean flow here being shown offset for visual clarity. Qualitative details of the instantaneous flow field can be seen to be well-approximated by the low-order regularized singularity representation. The magnitude of each flow field is given by the intensity of the shading, with projected streamlines shown in white.

The same general trend may be observed for virtual sperm in an above-below configuration, with speed shown as a solid curve, though reductions in swimming speed are much less in this instance. However, in contrast to side-by-side swimming, in the above-below configuration we observe a notable increase in linear speed when they are in very close proximity to one another, with separations of less than approximately $6.25 \mu \mathrm{m}$ giving rise to average speeds greater even than those attained by lone swimmers in the bulk. The magnitude of this effect increases further as separation is reduced, with an increase of around $4 \mu \mathrm{m} \mathrm{s}^{-1}$ over the bulk speed when at a separation of $1.5 \mu \mathrm{m}$, with swimmers nearing contact in this configuration. Thus, in addition to the aforementioned anisotropy of timescales, the effects of proximity on the speed of progression of the virtual spermatozoa are also configuration-dependent, in qualitative agreement with the results of Llopis et al. [20], though here observed accounting for the swimmer body and an idealized spermatozoan beating pattern. We observe that side-by-side swimming invariably results in reduced swimming speed, while close virtual spermatozoa in an above-below configuration have increased linear speed compared to both side-by-side swimmers and even lone cells in the bulk.

\section{E. Efficacy of PCA-derived singularity representations is limited in three-dimensional interactions}

We simulate the motion of a single swimmer in an unbounded domain to determine the swimmer's characteristic time-varying flow field, and form the PCA-derived singularity representation of the swimmer as described in Sec. IIE, with both flow fields and the accompanying regularized singularity representation being shown in Fig. 7. Simulating pairwise swimmer motion as in Ishimoto and Gaffney [41], we proceed to evaluate the accuracy of using such singularity representations for the simulation of swimmer interaction by repeating the analysis of Secs. III A, III B, and III D using this approximate framework.

First, we consider the predictions of this methodology with regard to separation-dependent swimming speed, reported above using the boundary element method as strongly dependent on swimmer configuration. With the results shown in Fig. 8(a) as blue curves, we observe that the singularity representation is unable to capture even the qualitative effects of swimmer proximity on swimming speed in the above-below configuration, highlighting a subtlety in the mechanism by which swimmers may mutually benefit from pairwise swimming. However, for side-by-side swimmers the concordance is fair, particularly as swimmer separation increases, suggesting a 


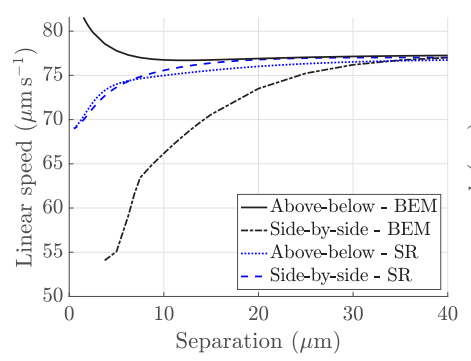

(a)

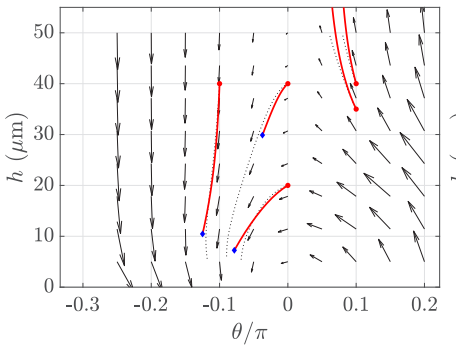

(b)

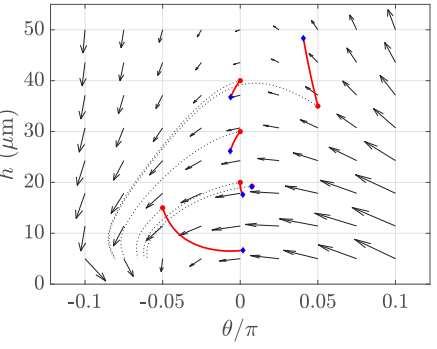

(c)

FIG. 8. Comparison of swimmer behaviors as predicted by the boundary element method and PCA-derived singularity representations. (a) The PCA-derived singularity representations (SR), shown here as blue curves, agree qualitatively with the boundary element method (BEM) predictions for linear swimming speed in the case of side-by-side swimming, though no such agreement is present in close proximity for swimmers in an above-below configuration. (b), (c) Evolution in $h-\theta$ space for (b) side-by-side and (c) above-below swimmers as predicted by singularity representations, shown as black arrows, with trajectories of the plane autonomous system shown as dotted black curves. Superimposed as solid red curves are projections of boundary element simulations onto the reduced space, with start and end points shown as red circles and blue diamonds, respectively. We note good agreement in the case of side-by-side swimming, though there is little correspondence between the predictions of the boundary element method and the singularity representations for swimmers in an above-below configuration. Levels of agreement are further evidenced by comparison with Figs. 3 and 4.

potential efficacy of this simple singularity representation in describing all but the closest of swimmer interactions in this configuration. Unsurprisingly, at large separations the cases of abovebelow and side-by-side converge to the free-space swimming speed of our virtual spermatozoa, as do the results of both the boundary element method and singularity representation computations, with swimmer-swimmer interactions decaying in magnitude as separation increases.

With reasonable agreement in swimming speed seen for side-by-side swimmers, we examine the long-time behaviors of pairwise swimmers in this configuration as in Sec. III A, instead using the PCA-derived singularity representations, simulating swimmer motion over a single beating period from various initial orientations and separations and forming the reduced dynamical system of Sec. II D. Shown in Fig. 8(b) are the dynamics in the $h-\theta$ space, with projections of long-time boundary element simulations superimposed as red curves for direct comparison. We observe very good qualitative agreement in overall swimmer behavior between the predictions of the boundary element method and the singularity representations, consistent with that noted for linear swimming speeds above. This consensus suggests that the singularity-based methodology provides a description of side-by-side dynamics that is capable of capturing qualitative swimmer behavior, and thus, may be widely applicable to studies of large-scale population interactions.

Now considering swimmers in an above-below configuration and repeating the construction of the approximate plane autonomous system as above, by drawing a comparison with Fig. 4 we see that the singularity representation fails to capture the characteristics of the motion as predicted by the boundary element method. Most notably, no stable swimming is present as a mode of pairwise swimming, with swimmers moving off into the bulk or colliding. Further, the overall dynamics of the reduced system computed via the singularity representation are not dissimilar to those predicted for side-by-side swimmers, suggesting that the anisotropy seen in boundary element simulations of our virtual spermatozoa is not captured by the low-order singularity representation.

Overall, we have seen good agreement between methodologies for swimmers in a side-by-side configuration, but little coherence in predicted behaviors for virtual spermatozoa in above-below configurations. Hence, we may conclude that the PCA-derived singularity representations should not be relied upon in their current form for the simulation of three-dimensional interactions in 
generality, though they provide remarkable qualitative agreement with computationally expensive high-accuracy methods for planar swimmers sharing a beating plane.

\section{DISCUSSION}

This study has considered the motion of two virtual spermatozoa in close proximity, restricted to planar beating and to two configurations of interest: side-by-side and above-below swimming. In doing so, we have identified a significant anisotropy in their relative motion, not simply limited to quantitative effects. Upon examining the linear swimming speed attained by our virtual, synchronized swimmers over a single beat period, we have observed that side-by-side spermatozoa make less progress along their respective headings than they would achieve in isolation. While the same, albeit substantially weaker, general trend may be noted in above-below swimmers at separations greater than approximately $10 \mu \mathrm{m}$, for reduced separations we note a remarkable increase in swimming speed, above that attained by lone swimmers in the bulk. We hypothesize that the pairwise attachment of spermatozoa in an above-below configuration may afford them a competitive advantage over nonbound individuals, with proximity increasing their speed of progression in line with the predictions of our computational model. In agreement with the predictions of the bead-and-spring model of Llopis et al. [20], our findings, however, are in part distinct from the similar computational results of Olson and Fauci [30] concerning side-by-side elastic filaments, which predict that swimming speeds increase with proximity for planar such filaments. This disparity in conclusions suggests that the inclusion of swimmer head geometry may have a significant impact on the predicted qualitative behaviors of the swimming pair, with care being necessitated in future theoretical works to accurately capture such effects.

Further anisotropy is present in the timescales of relative motion. We have seen that swimmers in an above-below scenario undergo greatly reduced changes to their configurations over a beating cycle when compared to the motion of swimmers sharing a beat plane. This slow evolution in relative position and direction for swimmers situated in an above-below configuration appears to be in approximate concordance with the general experimental observation that motile spermatozoa frequently appear to cross one another with little visible interaction, examples being found in the supplementary movies accompanying the recent works of Tung et al. [50] and Gadêlha et al. [51] concerning bovine and human spermatozoa, respectively. Further evidenced in the supplementary movies of Tung et al. [50], and additionally in those accompanying the work of Ishimoto et al. [40], are the limited effects of noise on swimming behaviors, with the effect of Brownian noise typically insignificant on the lengthscale of spermatozoa and synchronization persisting over reasonable timescales. However, thorough exploration of the impact of intra-spermatozoon variation on the pairwise behaviors reported in this work represents significant and pertinent future study, in addition to detailed investigations into the effects of background flows and confined geometries.

We have seen that the pairwise dynamics of virtual spermatozoa in side-by-side and above-below configurations may be described with notable accuracy by a plane autonomous system, motivating potential future consideration of pairwise interactions of further flagellated microswimmers in this way, extending in principle the approach of Ishikawa et al. [55], though here restricted to particular swimmer configurations due to the absence of symmetry. Most significantly, analysis of these reduced systems revealed a significant disparity between the long-time dynamics of above-below and side-by-side swimming, with the latter only exhibiting behaviors of collision and separation off into the bulk fluid. Consideration of above-below dynamics led to the identification of a remarkable mode of stable pairwise swimming in virtual spermatozoa, corresponding to a stable spiral in the plane autonomous system and whose existence was confirmed in silico by long-time simulation of a swimming pair. These behaviors are in partial agreement with general classical conclusions drawn from representing swimmers as a force dipole, valid in the far field for lone swimmers, which predicts that pushers like our virtual spermatozoa will generally swim facing one another [38], evidenced here by the noted reorientation of side-by-side swimmers. We have further seen that in fact near-field effects are significant in determining swimmer interactions, and indeed they 
give rise to a behavioral anisotropy that is not a feature of this most simple of far-field swimmer representations, with stable swimming only being observed here for above-below spermatozoa. The similar study of Llopis et al. [20] concerning active bead chains found that both above-below and side-by-side swimmers ultimately swim away from one another, further suggesting that the predicted interactions of nearby swimmers are sensitive to the inclusion or neglect of complex cellular morphology.

The existence of a stable swimming mode in silico raises the hypothesis that in vitro spermatozoa may swim stably as pairs even without adhering to one another, though the potentially complex effects of variation between individual swimmers are unclear and require detailed consideration. Indeed, throughout we have considered spermatozoa equipped with a synchronized kinematic description of their flagellar beating. Relaxation of this assumption necessitates careful treatment of the governing elastohydrodynamics and the internal molecular motor dynamics in order to fully capture the complex interactions between flow field and flagella, and in particular it is likely to be a topic of significant future study, as is the study of bound swimming sperm in the context of rodent sperm trains $[3,56]$.

Having simulated the behaviors of nearby swimmers using a high-accuracy boundary element method, we explored the qualitative accuracy of a simplified, coarse-grained approach for multiswimmer simulation, as implemented by Ishimoto and Gaffney [41]. We observed that this simplified approach is sufficient to capture qualitative features of side-by-side swimming, supporting its use in such circumstances. However, poor agreement between this methodology and the boundary element calculations was noted for above-below swimmers, with the singularity representation failing to reproduce the anisotropy associated with our planar beaters. This highlights the complexity of the near-field interactions of these flagellated swimmers, which are more intricate than is captured by our intuitive physical interpretation of the flow field via such a low-dimensional singularity representation, though differences between methodologies in predicted flow-field magnitude in the very near field, as seen in Fig. 7, may also contribute to the observed behavioral disparity. As the singularity method utilizes only regularized Stokeslets in its low-dimensional representations of the swimmers, we hypothesize that the inclusion of higher-order singularities may render enhanced anisotropy and improve its efficacy for simulating the motion of out-of plane swimming pairs, and is a potential direction for the refinement of this methodology. Without such an augmentation, the methodology of Ishimoto and Gaffney [41] thus, appears unreliable for three-dimensional pairwise interactions in general, though it is suitably accurate for in-plane interactions while affording significant computational simplicity and scalability to population-level models.

In summary, we have examined the behavioral consequences of hydrodynamic interactions between two synchronized flagellated swimmers moving in three dimensions, identifying a range of complex and subtle pairwise behaviors in virtual spermatozoa. Long-time simulations using highaccuracy boundary element simulations revealed diverse behavioral modes that are configurationdependent, with side-by-side swimming invariably resulting in collision or separation into the bulk. Throughout we have seen evidenced an anisotropy in the dynamics of nearby swimming, with proximity able to effect both increases and decreases in swimming speed depending on swimmer configuration, though such subtleties may not be captured by simulations using only low-order singularity representations of swimmers. Further, we have found a disparity in the timescales of relative motion between above-below and side-by-side swimming, and we have also noted that reductions to low-dimensional autonomous systems can well-describe pairwise swimmer dynamics. Finally, from analysis of such systems we have identified a remarkable swimming mode in synchronized virtual spermatozoa, one corresponding to stable pairwise swimming.

The research materials supporting this article have been made available [57].

\section{ACKNOWLEDGMENTS}

B.J.W. is supported by the UK Engineering and Physical Sciences Research Council (EPSRC), Grant No. EP/N509711/1. K.I. is supported by JSPS Overseas Research Fellowship (29-0146), 
MEXT Leading Initiative for Excellent Young Researchers (LEADER), and JSPS KAKENHI Grant No. JP18K13456.

\section{APPENDIX: PARAMETRIZATION OF THE VIRTUAL SPERMATOZOON HEAD}

With respect to the swimmer-fixed reference frame of spermatozoon $i \in\{1,2\}$, denoted $x_{i 1} x_{i 2} x_{i 3}$, as defined in the main text, we parametrize the swimmer head by length parameter $\xi \in\left[0, L_{\mathrm{h}}\right]$ and $\eta \in[0,2 \pi)$, where $L_{h}$ denotes the length of the virtual spermatozoon head, taken to be $4.5 \mu \mathrm{m}$. Adopting the idealized geometry of Smith et al. [14], the surface of the spermatozoon head is parametrized by $\left(x_{i 1}, x_{i 2}, x_{i 3}\right)=(-\xi, r \sin \eta, r \cos \eta)$, where $r$ is given in terms of $\xi$ and $\eta$ by

$$
\begin{gathered}
r^{2}=\frac{1-\left(2 \xi / L_{h}-1\right)^{2}}{\beta_{1}^{2} \sin \eta^{2}+\beta_{2}^{2} \cos \eta^{2}} \beta_{1}^{2} \beta_{2}^{2}, \\
\beta_{1}=r_{1}-\hat{r} \sin \left(4 \xi / L_{h}-2\right), \\
\beta_{2}=r_{2}+\hat{r} \sin \left(4 \xi / L_{h}-2\right) .
\end{gathered}
$$

We specify the constant morphological parameters as $r_{1}=0.5 \mu \mathrm{m}, r_{2}=1.25 \mu \mathrm{m}$, and $\hat{r}=$ $0.28 \mu \mathrm{m}$, representing a model human spermatozoon following Ishimoto and Gaffney [10]. Such parameters yield ellipsoidal cross sections with maximal major and minor axes of approximately 2.68 and $1.28 \mu \mathrm{m}$, respectively, as shown in Fig. 1(a).

[1] J. Gray, Ciliary Movement (Cambridge University Press, Cambridge, England, 1928).

[2] L. Rothschild, Measurement of sperm activity before artificial insemination, Nature (London) $\mathbf{1 6 3}, 358$ (1949).

[3] H. Moore, K. Dvoráková, N. Jenkins, and W. Breed, Exceptional sperm cooperation in the wood mouse, Nature (London) 418, 174 (2002).

[4] A. Creppy, O. Praud, X. Druart, P. L. Kohnke, and F. Plouraboué, Turbulence of swarming sperm, Phys. Rev. E 92, 032722 (2015).

[5] M. J. Zinaman, C. C. Brown, S. G. Selevan, and E. D. Clegg, Semen quality and human fertility: A prospective study with healthy couples, J. Androl. 21, 145 (2000).

[6] D. Lai, G. D. Smith, and S. Takayama, Lab-on-a-chip biophotonics: Its application to assisted reproductive technologies, J. Biophoton. 5, 650 (2012).

[7] J. E. Swain, D. Lai, S. Takayama, and G. D. Smith, Thinking big by thinking small: Application of microfluidic technology to improve ART, Lab Chip 13, 1213 (2013).

[8] C.-K. Tung, F. Ardon, A. G. Fiore, S. S. Suarez, and M. Wu, Cooperative roles of biological flow and surface topography in guiding sperm migration revealed by a microfluidic model, Lab Chip 14, 1348 (2014).

[9] K. Ishimoto and E. A. Gaffney, Fluid flow and sperm guidance: A simulation study of hydrodynamic sperm rheotaxis, J. R. Soc. Interface 12, 20150172 (2015).

[10] K. Ishimoto and E. A. Gaffney, A study of spermatozoan swimming stability near a surface, J. Theor. Biol. 360, 187 (2014).

[11] B. M. Friedrich, I. H. Riedel-Kruse, J. Howard, and F. Julicher, High-precision tracking of sperm swimming fine structure provides strong test of resistive force theory, J. Exp. Biol. 213, 1226 (2010).

[12] J. Elgeti, U. B. Kaupp, and G. Gompper, Hydrodynamics of sperm cells near surfaces, Biophys. J. 99, 1018 (2010).

[13] L. J. Fauci and A. McDonald, Sperm motility in the presence of boundaries, Bull. Math. Biol. 57, 679 (1995). 
[14] D. J. Smith, E. A. Gaffney, J. R. Blake, and J. C. Kirkman-Brown, Human sperm accumulation near surfaces: A simulation study, J. Fluid Mech. 621, 289 (2009).

[15] S. F. Schoeller and E. E. Keaveny, From flagellar undulations to collective motion: Predicting the dynamics of sperm suspensions, J. R. Soc. Interface 15, 20170834 (2018).

[16] Y. Yang, V. Marceau, and G. Gompper, Swarm behavior of self-propelled rods and swimming flagella, Phys. Rev. E 82, 031904 (2010).

[17] G. Taylor, Analysis of the swimming of microscopic organisms, Proc. R. Soc. London, Ser. A 209, 447 (1951).

[18] Y. Yang, J. Elgeti, and G. Gompper, Cooperation of sperm in two dimensions: Synchronization, attraction, and aggregation through hydrodynamic interactions, Phys. Rev. E 78, 061903 (2008).

[19] J. Simons, L. Fauci, and R. Cortez, A fully three-dimensional model of the interaction of driven elastic filaments in a stokes flow with applications to sperm motility, J. Biomech. 48, 1639 (2015).

[20] I. Llopis, I. Pagonabarraga, M. Cosentino Lagomarsino, and C. P. Lowe, Cooperative motion of intrinsic and actuated semiflexible swimmers, Phys. Rev. E 87, 032720 (2013).

[21] K. Ishimoto and E. A. Gaffney, Squirmer dynamics near a boundary, Phys. Rev. E 88, 062702 (2013).

[22] B. J. Walker, R. J. Wheeler, K. Ishimoto, and E. A. Gaffney, Boundary behaviours of Leishmania mexicana: A hydrodynamic simulation study, J. Theor. Biol. 462, 311 (2019).

[23] D. M. Woolley, R. F. Crockett, W. D. I. Groom, and S. G. Revell, A study of synchronisation between the flagella of bull spermatozoa, with related observations, J. Exp. Biol. 212, 2215 (2009).

[24] D. R. Brumley, K. Y. Wan, M. Polin, and R. E. Goldstein, Flagellar synchronization through direct hydrodynamic interactions, eLife 3, 1 (2014).

[25] B. M. Friedrich and I. H. Riedel-Kruse, Flagellar beating: Row with the flow, eLife 3, e03804 (2014).

[26] N. Uchida, R. Golestanian, and R. R. Bennett, Synchronization and collective dynamics of flagella and cilia as hydrodynamically coupled oscillators, J. Phys. Soc. Jpn. 86, 101007 (2017).

[27] G. J. Elfring and E. Lauga, Passive hydrodynamic synchronization of two-dimensional swimming cells, Phys. Fluids 23, 011902 (2011).

[28] R. E. Goldstein, E. Lauga, A. I. Pesci, and M. R. E. Proctor, Elastohydrodynamic synchronization of adjacent beating flagella, Phys. Rev. Fluids 1, 073201 (2016).

[29] L. J. Fauci, Interaction of oscillating filaments: A computational study, J. Comput. Phys. 86, 294 (1990).

[30] S. D. Olson and L. J. Fauci, Hydrodynamic interactions of sheets vs filaments: Synchronization, attraction, and alignment, Phys. Fluids 27, 121901 (2015).

[31] A. Sokolov and I. S. Aranson, Physical Properties of Collective Motion in Suspensions of Bacteria, Phys. Rev. Lett. 109, 248109 (2012).

[32] A. Zöttl and H. Stark, Hydrodynamics Determines Collective Motion and Phase Behavior of Active Colloids in Quasi-Two-Dimensional Confinement, Phys. Rev. Lett. 112, 118101 (2014).

[33] D. Saintillan and M. J. Shelley, Orientational Order and Instabilities in Suspensions of Self-Locomoting Rods, Phys. Rev. Lett. 99, 058102 (2007).

[34] N. Oyama, J. J. Molina, and R. Yamamoto, Purely hydrodynamic origin for swarming of swimming particles, Phys. Rev. E 93, 043114 (2016).

[35] G. Li and A. M. Ardekani, Collective Motion of Microorganisms in a Viscoelastic Fluid, Phys. Rev. Lett. 117, 118001 (2016).

[36] B. Ezhilan, M. J. Shelley, and D. Saintillan, Instabilities and nonlinear dynamics of concentrated active suspensions, Phys. Fluids 25, 070607 (2013).

[37] M. C. Marchetti, J. F. Joanny, S. Ramaswamy, T. B. Liverpool, J. Prost, M. Rao, and R. A. Simha, Hydrodynamics of soft active matter, Rev. Mod. Phys. 85, 1143 (2013).

[38] E. Lauga and T. R. Powers, The hydrodynamics of swimming microorganisms, Rep. Prog. Phys. 72 , 096601 (2009).

[39] C. M. Pooley, G. P. Alexander, and J. M. Yeomans, Hydrodynamic Interaction Between Two Swimmers at Low Reynolds Number, Phys. Rev. Lett. 99, 228103 (2007).

[40] K. Ishimoto, H. Gadêlha, E. A. Gaffney, D. J. Smith, and J. Kirkman-Brown, Coarse-Graining the Fluid Flow Around a Human Sperm, Phys. Rev. Lett. 118, 124501 (2017). 
[41] K. Ishimoto and E. A. Gaffney, Hydrodynamic clustering of human sperm in viscoelastic fluids, Sci. Rep. 8, 15600 (2018).

[42] R. Cortez, The method of regularized stokeslets, SIAM J. Sci. Comput. 23, 1204 (2001).

[43] J. Cummins and P. Woodall, On mammalian sperm dimensions, J. Reprod. Fertil. 75, 153 (1985).

[44] R. D. Dresdner and D. F. Katz, Relationships of mammalian sperm motility and morphology to hydrodynamic aspects of cell function, Biol. Reprod. 25, 920 (1981).

[45] D. J. Smith, E. A. Gaffney, H. Gadêlha, N. Kapur, and J. C. Kirkman-Brown, Bend propagation in the flagella of migrating human sperm, and its modulation by viscosity, Cell Motil. Cytoskeleton 66, 220 (2009).

[46] C. Pozrikidis, Boundary Integral and Singularity Methods for Linearized Viscous Flows (Cambridge University Press, Cambridge, 1992).

[47] C. Pozrikidis, A Practical Guide to Boundary Element Methods with the Software Library BEMLIB (CRC, Boca Raton, FL, 2002).

[48] J. D. Klein, A. R. Clapp, and R. B. Dickinson, Direct measurement of interaction forces between a single bacterium and a flat plate, J. Colloid Interface Sci. 261, 379 (2003).

[49] K. Ishimoto and E. A. Gaffney, Mechanical tuning of mammalian sperm behaviour by hyperactivation, rheology and substrate adhesion: A numerical exploration, J. R. Soc. Interface 13, 20160633 (2016).

[50] C.-K. Tung, C. Lin, B. Harvey, A. G. Fiore, F. Ardon, M. Wu, and S. S. Suarez, Fluid viscoelasticity promotes collective swimming of sperm, Sci. Rep. 7, 3152 (2017).

[51] H. Gadêlha, E. A. Gaffney, D. J. Smith, and J. C. Kirkman-Brown, Nonlinear instability in flagellar dynamics: A novel modulation mechanism in sperm migration? J. R. Soc. Interface 7, 1689 (2010).

[52] B. J. Walker, K. Ishimoto, R. J. Wheeler, and E. A. Gaffney, Response of monoflagellate pullers to a shearing flow: A simulation study of microswimmer guidance, Phys. Rev. E 98, 063111 (2018).

[53] K. Ishimoto, H. Gadêlha, E. A. Gaffney, D. J. Smith, and J. Kirkman-Brown, Human sperm swimming in a high viscosity mucus analogue, J. Theor. Biol. 446, 1 (2018).

[54] B. Chakrabarti and D. Saintillan, Spontaneous oscillations, beating patterns, and hydrodynamics of active microfilaments, Phys. Rev. Fluids 4, 043102 (2019).

[55] T. Ishikawa, M. P. Simmonds, and T. J. Pedley, Hydrodynamic interaction of two swimming model microorganisms, J. Fluid Mech. 568, 119 (2006).

[56] H. S. Fisher and H. E. Hoekstra, Competition drives cooperation among closely related sperm of deer mice, Nature (London) 463, 801 (2010).

[57] B. J. Walker, K. Ishimoto, and E. A. Gaffney, Data relating to "Pairwise hydrodynamic interactions of synchronized spermatozoa", https://doi.org/10.5287/bodleian:R5ppOao4z (2019). 